Chapter 4

\title{
An Ant Colony Optimization Algorithm for Area Traffic Control
}

\author{
Soner Haldenbilen, Ozgur Baskan and Cenk Ozan
}

Additional information is available at the end of the chapter

http://dx.doi.org/10.5772/51695

\section{Introduction}

The optimization of traffic signal control is at the heart of urban traffic control. Traffic signal control which encloses delay, queuing, pollution, fuel consumption is a multi-objective optimization. For a signal-controlled road network, using the optimization techniques in determining signal timings has been discussed greatly for decades. Due to complexity of the Area Traffic Control (ATC) problem, new methods and approaches are needed to improve efficiency of signal control in a signalized road network. In urban networks, traffic signals are used to control vehicle movements so as to reduce congestion, improve safety, and enable specific strategies such as minimizing delays, improving environmental pollution, etc. [1]. Signal systems that control road junctions are operated according to the type of junction. Although the optimization of signal timings for an isolated junction is relatively easy, the optimization of signal timings in coordinated road networks requires further research due to the "offset" term. Early methods such as that of [2] only considered an isolated signalized junction. Later, fixed time strategies were developed that optimizing a group of signalized junctions using historical flow data [3]. For the ATC, TRANSYT-7F is one of the most useful network study software tools for optimizing signal timing and also the most widely used program of its type. It consists of two main parts: A traffic flow model and a signal timing optimizer. Traffic model utilizes a platoon dispersion algorithm that simulates the normal dispersion of platoons as they travel downstream. It simulates traffic in a network of signalized intersections to produce a cyclic flow profile of arrivals at each intersection that is used to compute a Performance Index $(P I)$ for a given signal timing and staging plan. The PI in TRANSYT-7F may be defined in a number of ways. One of the TRANSYT-7F's PI is the Disutility Index $(D I)$. The DI is a measure of disadvantageous operation; that is stops, delay, fuel consumption, etc. Optimization in TRANSYT-7F consists of a series of trial simulation 
runs, using the TRANSYT-7F simulation engine. Each simulation run is assigned a unique signal timing plan by the optimization processor. The optimizer applies the Hill-Climbing (HC) or Genetic Algorithm (GA) searching strategies. The trial simulation run resulting in the best performance is reported as optimal. Although the GA is mathematically better suited for determining the absolute or global optimal solution, relative to HC optimization, it generally requires longer program running times, relative to HC optimization [4].

This chapter proposes Ant Colony Optimization (ACO) based algorithm called ACORSES proposed by [5] for finding optimum signal parameters in coordinated signalized networks for given fixed set of link flows. The ACO is the one of the most recent techniques for approximate optimization methods. The main idea is that it is indirect local communication among the individuals of a population of artificial ants. The core of ant's behaviour is the communication between the ants by means of chemical pheromone trails, which enables them to find shortest paths between their nest and food sources. This behaviour of real ant colonies is exploited to solve optimization problems. The proposed algorithm is based on each ant searches only around the best solution of the previous iteration with reduced search space. It is proposed for improving ACO's solution performance to reach global optimum fairly quickly. In this study, for solving the ATC problem, Ant Colony Optimization TRANSYT (ACOTRANS) model is developed. TRANSYT-7F traffic model is used to estimate total network PI.

Wong (1995) proposed group-based optimization of signal timings for area traffic control. In addition, the optimization of signal timings for ATC using group-based control variables was proposed by [7]. However, it was reported that obtaining the derivations of the PI for each of the control variable was mathematically difficult. Heydecker (1996) decomposed the optimization of traffic signal timings into two levels; first, optimizing the signal timings at the individual junction level using the group-based approach, and second, combining the results from individual junction level with network level decision variables such as offset and common cycle time. Wong et al. (2000) developed a time-dependent TRANSYT traffic model for the evaluation of PI. It was found that the time-dependent model produces a reasonable estimate of PI for under saturated to moderately oversaturated conditions. Wong et al. (2002) developed a time-dependent TRANSYT traffic model which is a weighted combination of the estimated delay and number of stops. A remarkable improvement over the average flow scenario was obtained and when compared with the signal plans from independent analyses, a good improvement was found. Girianna and Benekohal (2002) presented two different GA techniques which are applied on signal coordination for oversaturated networks. Signal coordination was formulated as a dynamic optimization problem and is solved using GA for the entire duration of congestion.

Similarly, Ceylan (2006) developed a GA with TRANSYT-HC optimization routine, and proposed a method for decreasing the search space to solve the ATC problem. Proposed approach is better than signal timing optimization regarding optimal values of timings and PI when it is compared with TRANSYT. Chen and Xu (2006) investigated the application of Particle Swarm Optimization (PSO) algorithm to solve signal timing optimization problem. Their results showed that PSO can be applied to the traffic signal timing optimization prob- 
lem under different traffic demands. A hybrid optimization algorithm for simultaneously solving delay-minimizing and capacity-maximizing ATC was presented by [14]. Numerical computations and comparisons were conducted on a variety of road networks. Numerical tests showed that the effectiveness and robustness of this hybrid heuristic algorithm. Similarly, Chiou (2007) presented a computation algorithm based on the projected Quasi-Newton method to effectively solve the ATC problem. The proposed method combining the locally optimal search and global search heuristic achieved substantially better performance than did traditional approaches in solving the ATC problem with expansions of link capacity.

Dan and Xiaohong (2008) developed a real-coded improved GA with microscopic traffic simulation model to find optimal signal plans for ATC problem, which takes the coordination of signals timing for all signal-controlled junction into account. The results showed that the method based on GA could minimize delay time and improve capacity of network. Li (2011) presented an arterial signal optimization model that consider queue blockage among intersection lane groups under oversaturated conditions. The proposed model captures traffic dynamics with the cell transmission concept, which takes into account complex flow interactions among different lane groups. Through comparisons with signal-timing plans from TRANSYT-7F, the model was successful for signal-timing optimization particularly under congested conditions. The optimization of signal timings on coordinated signalized road network, which includes a set of non-linear mathematical formulations, is very difficult. Therefore, new methods and approaches are needed to improve efficiency of signal control in a road network due to complexity of the ATC problem. Although there are many studies in literature with different heuristic methods to optimize traffic signal timings, there is no application of ACO to this area. Thus, this study proposes Ant Colony Optimization TRANSYT-7F (ACOTRANS) model in which ACO and TRANSYT-7F are combined for solving the ATC problem. The remaining content of this chapter is organized as follows. ACO algorithm and its solution process are given in Section 2, and definition of the ACOTRANS model is provided in Section 3. Numerical application is presented in Section 4. Last section is about the conclusions.

\section{Ant Colony Optimization}

Ant algorithms were inspired by the observation of real ant colonies. Ants are social insects that live in colonies and whose behaviour is directed more to the survival of the colony as a whole than to that of a single individual component of the colony. Social insects have captured the attention of many scientists because of the high structuration level their colonies can achieve, especially when compared to the relative simplicity of the colony's individuals. An important and interesting behaviour of ant colonies is their foraging behaviour, and, in particular, how ants can find shortest paths between food sources and their nest [18]. Ants are capable of finding the shortest path from food source to their nest or vice versa by smelling pheromones which are chemical substances they leave on the ground while walking. Each ant probabilistically prefers to follow a direction rich in pheromone. This behaviour of real ants can be used to explain how they can find a shortest path [19]. The main idea is that 
it is indirect local communication among the individuals of a population of artificial ants. The core of ant's behavior is the communication between the ants by means of chemical pheromone trails, which enables them to find shortest paths between their nest and food sources. This behaviour of real ant colonies is exploited to solve optimization problems [20]. The general ACO algorithm is illustrated in Fig. 1. The first step consists mainly on the initialization of the pheromone trail. At beginning, each ant builds a complete solution to the problem according to a probabilistic state transition rules. They depend mainly on the state of the pheromone.

\begin{tabular}{|ll|}
\hline Step 1: & $\begin{array}{l}\text { Initialize } \\
\text { Pheromone trail }\end{array}$ \\
Step 2: & $\begin{array}{l}\text { Iteration } \\
\text { Repeat for each ant } \\
\text { Solution construction using pheromone trail } \\
\text { Update the pheromone trail } \\
\text { Until stopping criteria }\end{array}$ \\
\hline
\end{tabular}

Figure 1. A generic ant algorithm.

Once all ants generate a solution, then global pheromone updating rule is applied in two phases; an evaporation phase, where a fraction of the pheromone evaporates, and a reinforcement phase, where each ant deposits an amount of pheromone which is proportional to the fitness. This process is repeated until stopping criteria is met. The ACORSES proposed by [5] is consisted of three main phases; Initialization, pheromone update and solution phase. All of these phases build a complete search to the global optimum as can be seen in Fig. 2.

As shown in Figure 2, pheromone update phase is located after the initialization phase, means that quantity of pheromone intensifies at each iteration within the reduced search space. Thus, global optimum is searched within the reduced search space using best values obtained from new ant colony in the previous iteration. Main advantageous of the ACORSES is that Feasible Search Space (FSS) is reduced with $\beta$ and it uses the information taken from previous iteration.

At the beginning of the first cycle, all ants search randomly to the best solution of a given problem within the FSS, and old ant colony is created at initialization phase. After that, quantity of pheromone is updated. In the solution phase, new ant colony is created based on the best solution from the old ant colony using Equation (1) and (2). Then, the best solutions of two colonies are compared. At the end of the first cycle, FSS is reduced by $\beta$ and best solution obtained from the previous iteration is kept. Global or near global optimum solution is then searched in the reduced search space during the solution progress. The ACORSES reaches to the global or near global optimum as ants find their routes in the limited space [5]. 


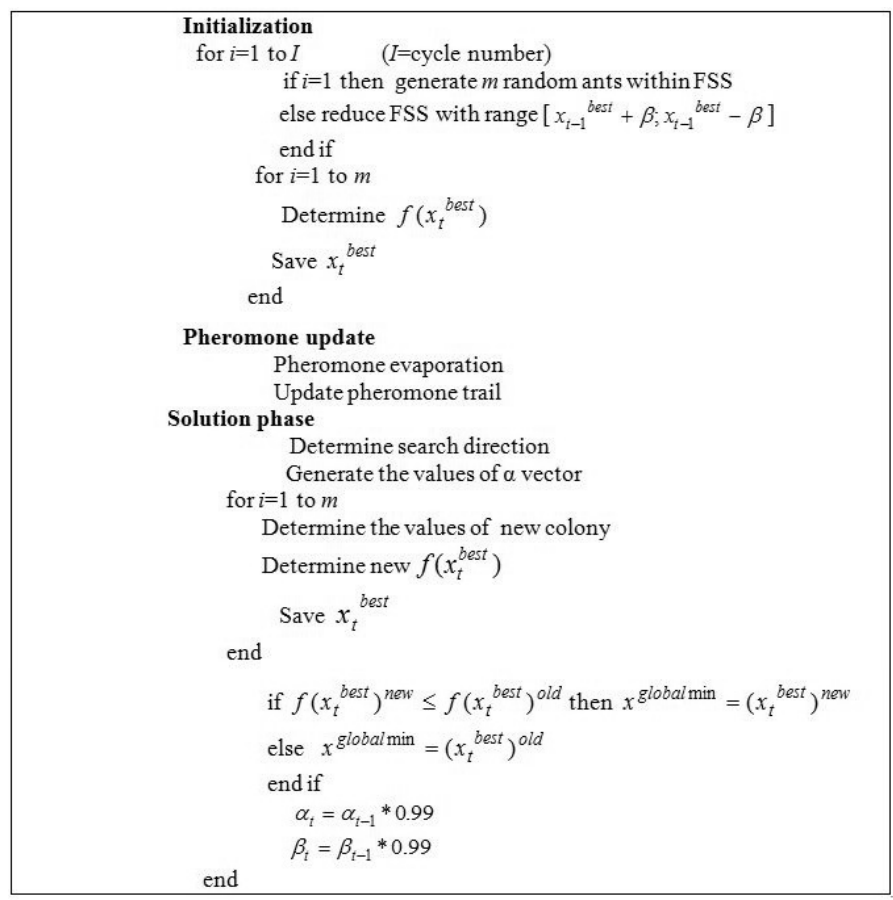

Figure 2. Steps of ACORSES [5].

Let number of $m$ ants being associated with $m$ random initial vectors $\left(x^{k}, k=1,2,3, \ldots \ldots m\right)$. The solution vector of each ant is updated using following expression:

$$
\begin{aligned}
& x_{t}^{k(\text { new })}=x_{t}^{k(\text { old })} \pm \alpha \\
& (t=1,2, \ldots . ., I)
\end{aligned}
$$

where $x_{t}^{k(n e w)}$ is the solution vector of the $k^{\text {th }}$ ant at cycle $t, x_{t}^{k(o l d)}$ is the solution obtained from the previous step at cycle $t$, and $\alpha$ is a vector generated randomly to determine the length of jump. $\alpha$ controls the global optimum search direction not being trapped at bad local optimum. Ant vector $x_{t}^{k(n e w)}$ obtained at $t^{\text {th }}$ cycle in (1) is determined using the value of same ant obtained from previous step. Furthermore, in expression (1), (+) sign is used when point $x_{t}^{k}$ is on the left of the best solution on the $x$ coordinate axis. (-) sign is used when point $x_{t}^{k}$ is on the right of the best solution on the same axis. The direction of search is defined by expression (2). 


$$
\bar{x}_{t}^{\text {best }}=x_{t}^{\text {best }}+\left(x_{t}^{\text {best }} * 0.01\right)
$$

If $f\left(\bar{x}_{t}{ }^{\text {best }}\right) \leq f\left(x_{t}{ }^{\text {best }}\right),(+)$ sign is used in (1). Otherwise, $(-)$ sign is used. $( \pm)$ sign defines the search direction to reach to the global optimum. $\alpha$ value is used to define the length of jump, and it will be gradually decreased in order not to pass over global optimum, as shown in Fig. 2. At the end of each cycle, a new ant colony is developed as the number of ants generated in old colony. Quantity of pheromone $\left(\tau_{t}\right)$ is reduced to simulate the evaporation process of real ant colonies using (3) in the pheromone update phase. After reducing of the number of pheromone, it is updated using (4). Quantity of pheromone only intensifies around the best objective function value. This process is repeated until the given number of cycle, $I$, is completed. Initial pheromone intensity is set to the value of 100 .

$$
\begin{gathered}
\tau_{t}=0.1 * \tau_{t-1} \\
\tau_{t}=\tau_{t-1}+0.01 * f\left(x_{t-1}^{\text {best }}\right)
\end{gathered}
$$

ACO uses real numbers instead of coding them as in GA to optimise any given objective function. This is one of the main advantage of ACO that it provides to optimise the signal timings with less mathematically lengthy. Moreover, ACORSES algorithm has ability to reach to the global optimum quickly without being trapped in bad local optimum because it uses the reduced search space and the values of optimum signal timings are then searched in the reduced search space during the algorithm progress. The ACORSES reaches to the global optimum or near global optimum as ants find their routes in the limited space. For better understanding, consider a problem of five ants represents the formulation of the problem.

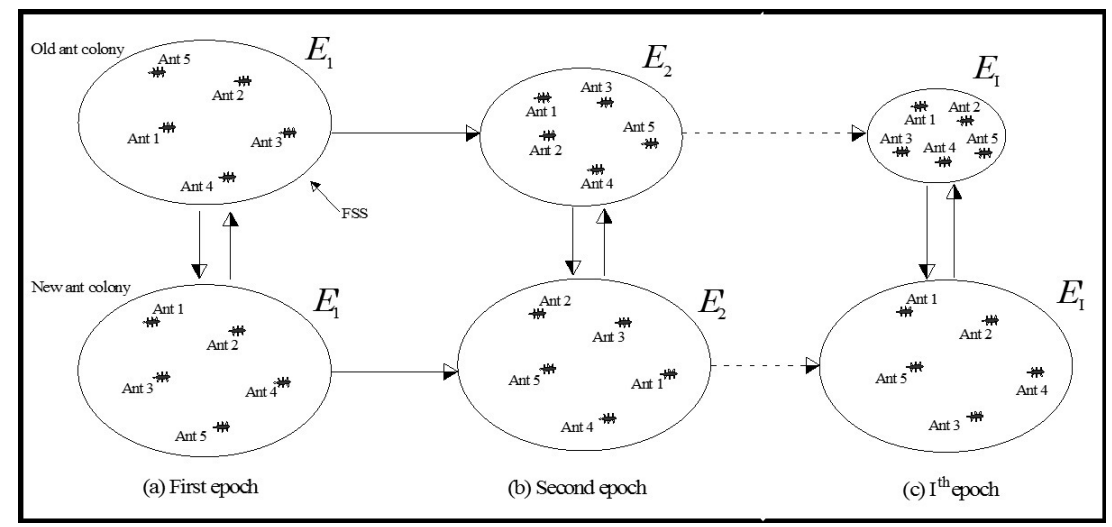

Figure 3. Main idea of the ACORSES [5] 
As shown in Fig.3, five ants being associated five random initial vectors. At the beginning of the first cycle (Fig. 3a), old ant colony is randomly created within the feasible search space for any given problem. After pheromone update phase, new ant colony is created at the last phase of the first cycle according to old ant colony using Equation (1) and (2). After that, the best values of the two colonies are compared. According to the best value obtained so far by comparing the old and new colonies and $\beta$, the FSS is reduced at the beginning of the second cycle and once again old ant colony is created, as can be seen in Fig. 3b. The new ant colony is created at the last phase of the second cycle according to randomly generated $\alpha$ value using Equation (1). Any of the newly created solution vectors may be outside the reduced search space that is created at the beginning of the second cycle. Therefore, created new ant colony prevents being trapped in bad local optimum [5].

\section{ACOTRANS for area traffic control}

The ACOTRANS consists of two main parts namely ACO based algorithm and TRANSYT-7F traffic model. ACO algorithm optimizes traffic signal timings under fixed set of link flows. TRANSYT-7F traffic model is used to compute PI, which is called objective function, for a given signal timing and staging plan in network. The network Disutility Index $(D I)$, one of the TRANSYT-7F's PI, is used as objective function. The DI is a measure of disadvantageous operation; that is stops, delay, fuel consumption, etc. The standard TRANSYT-7F's $D I$ is linear combination of delay and stops. The objective function and corresponding constraints are given in Eq. (5).

$$
P I=\underset{\psi, \mathbf{q}=\text { fixed }}{\operatorname{Min} D I}=\sum_{a \in L}\left[w_{d_{a}} \cdot d_{a}(\psi)+K \cdot w_{s_{a}} \cdot S_{a}(\psi)\right]
$$

Subject to $\psi(c, \theta, \varphi) \in \Omega_{0} ;\left(\begin{array}{ll}c_{\min } \leq c \leq c_{\max } & \text { cycle time constraints } \\ 0 \leq \theta \leq c & \text { values of offset constraints } \\ \varphi_{\min } \leq \varphi \leq c & \text { green time constraints } \\ \sum_{i=1}^{z}(\varphi+I)_{i}=c & \end{array}\right)$

where $d_{a}$ is delay on link $a$ ( $L$ set of links), $w_{d_{a}}$ is link-specific weighting factor for delay $d, K$ is stop penalty factor to express the importance of stops relative to delay, $S_{a}$ is stop on link $a$ per second, $w_{s_{a}}$ is link-specific weighting factor for stops $S$ on link $a$, q is fixed set of link flows, $\psi$ is signal setting parameters, $c$ is common cycle time (sec), $\theta$ is offset time (sec), $\varphi$ is green time (sec), $\Omega_{0}$ is feasible region for signal timings, $I$ is intergreen time (sec), and $z$ is number of stages at each signalized intersection in a given road network.

The green timings can be distributed to all signal stages in a road network according to Eq. (6) in order to provide the cycle time constraint [21]. 


$$
\begin{aligned}
& \varphi_{i}=\varphi_{\min , i}+\frac{p_{i}}{\sum_{k=1}^{z} p_{i}}\left(c-\sum_{k=1}^{z} I_{k}-\sum_{k=1}^{z} \varphi_{\min , k}\right) \\
& i=1,2, \ldots . . z
\end{aligned}
$$

where $\varphi_{i}$ is the green time (sec) for stage $i, \varphi_{\min , i}$ is minimum green time (sec) for stage $i, p_{i}$ is generated randomly green timings (sec) for stage $i, z$ is the number of stages and $I$ is intergreen time (sec) between signal stages and $c$ is the common cycle time of the network (sec).

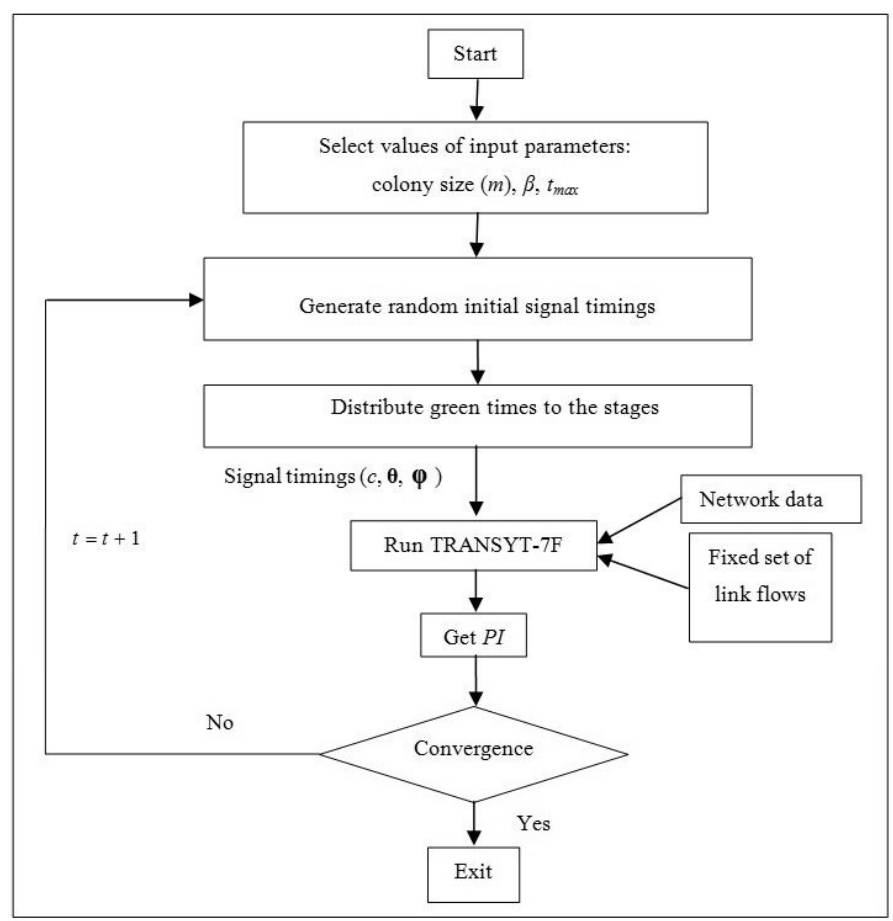

Figure 4. The flowchart of the ACOTRANS.

In the ACOTRANS, optimization steps can be given in the following way:

Step 0: Initialization. Define the user specified parameters; the number of decision variables $(n)$ (this number is sum of the number of green times as stage numbers at each intersection, the number of offset times as intersection numbers and common cycle time), the constraints for each decision variable, the size of ant colony $(m)$, search space value $(\beta)$ for each decision variable.

Step 1: Set $t=1$. 
Step 2: Generate the random initial signal timings, $\psi(c, \theta, \varphi)$ within the constraints of decision variables.

Step 3: Distribute to the initial green timings to the stages according to distribution rule as mentioned above. At this step, randomly generated green timings at Step 2 are distributed to the stages according to generated cycle time at the same step, minimum green and intergreen time.

Step 4: Get the network data and fixed set of link flows for TRANSYT-7F traffic model.

Step 5: Run TRANSYT-7F.

Step 6: Get the network PI. At this step, the PI is determined using TRANSYT-7F traffic model.

Step 7: If $t=t_{\max }$ then terminate the algorithm; otherwise, $t=t+1$ and go to Step 2.

The flowchart of the ACOTRANS can be seen in Fig. (4).

\section{Numerical Application}

The ACOTRANS is tested on two example networks taken from literature. First, it is applied to two junction road network. The network contains one origin destination pair, eight links and six signal setting variables. The network and its representation of signal stages can be seen in Fig. (5a) and (5b). The fixed set of link flows, taken from [22] is given in Table 1.
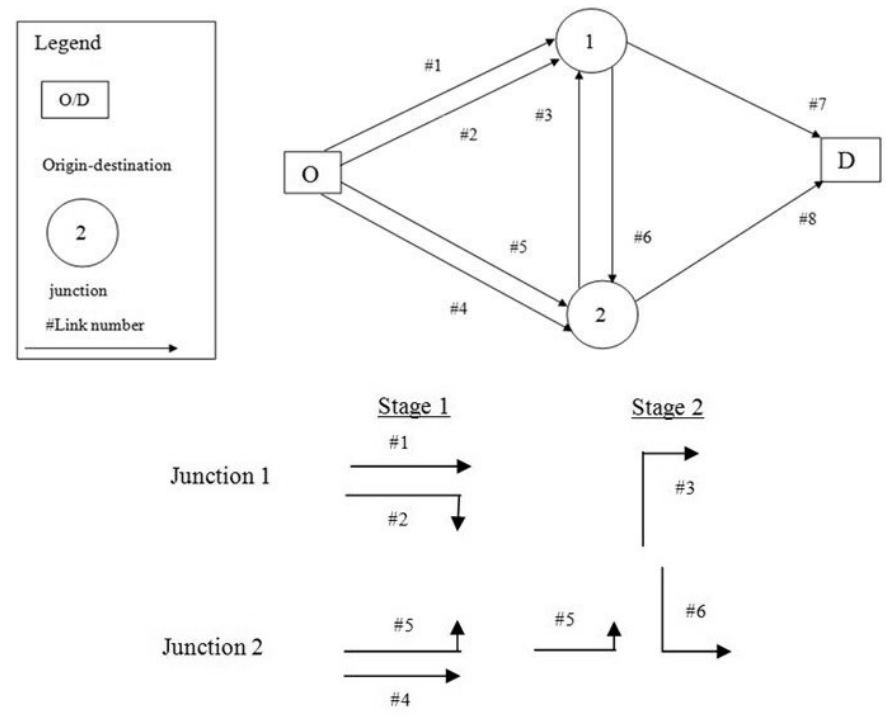

Figure 5. a) Two junction network; b) Representation of signal stages of two-junction network. 


\begin{tabular}{cccc}
\hline $\begin{array}{c}\text { Link } \\
\text { number }\end{array}$ & $\begin{array}{c}\text { Link } \\
\text { flow } \\
\text { (veh/h) }\end{array}$ & $\begin{array}{c}\text { Saturation } \\
\text { flow } \\
\text { (veh/h) }\end{array}$ & $\begin{array}{c}\text { Free-flow } \\
\text { travel time } \\
\text { (sec) }\end{array}$ \\
\hline 1 & 615 & 1800 & 20 \\
\hline 2 & 45 & 1800 & 20 \\
\hline 3 & 225 & 1800 & 20 \\
\hline 4 & 615 & 1800 & 20 \\
\hline 5 & 225 & 1800 & 20 \\
\hline 6 & 45 & 1800 & 20
\end{tabular}

Table 1. Fixed set of link flows on two junction network.

The constraints on signal timings are set as follows:

$36 \leq c \leq 90$ cycle time constraint

$0 \leq \theta \leq c$ offsets

$7 \leq \varphi \leq$ cgreen split

$I_{1-2}=I_{2-1}=5$ seconds intergreen time

The ACOTRANS model was coded by the MATLAB software. It is performed with the following user-specified parameters: colony size is 20 , and maximum number of cycle $\left(t_{\max }\right)$ is 75. The convergence of the model can be seen in Fig. (6).

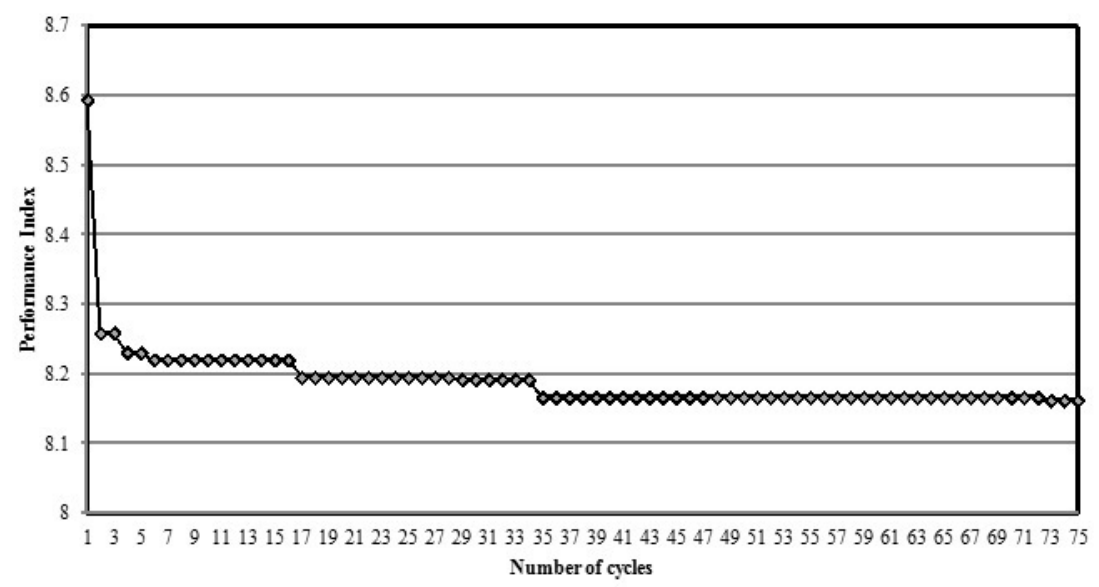

Figure 6. The convergence of the ACOTRANS for small sized network. 
In $75^{\text {th }}$ cycle, ACOTRANS is reached to PI value of 8.16. The common network cycle time obtained from the ACOTRANS is 76 sec. In addition, two junction road network is optimized using TRANSYT-7F which included GA and HC optimization tools. In GA parameters, population size and maximum number of cycle are chosen 20 and 300, respectively. In HC optimization tool in TRANSYT-7F, the default optimization parameters used by program are effective and system is simulated for every integer cycle length between minimum and maximum cycle length. Therefore, HC optimization parameters are not being manipulated. For two junction road network, the ACOTRANS model and TRANSYT-7F optimizers' results are given in Table 2 .

\begin{tabular}{|c|c|c|c|c|c|c|}
\hline & \multirow{2}{*}{$\begin{array}{c}\text { Performance } \\
\text { Index }\end{array}$} & \multirow{2}{*}{$\begin{array}{l}\text { Cycle } \\
\text { Time } \\
\text { c(s) }\end{array}$} & \multirow{2}{*}{$\begin{array}{c}\text { Junction } \\
\text { number } \\
i\end{array}$} & \multicolumn{2}{|c|}{ Duration of stages (s) } & \multirow{2}{*}{$\begin{array}{c}\text { Offsets } \\
\text { (s) } \\
I_{1-2}=I_{2-1}=5\end{array}$} \\
\hline & & & & $\begin{array}{c}\text { Stage } 1 \\
\theta_{i}\end{array}$ & $\begin{array}{c}\text { Stage } 2 \\
\varphi_{i, 1}\end{array}$ & \\
\hline \multirow{2}{*}{ ACOTRANS } & \multirow{2}{*}{8.16} & \multirow{2}{*}{76} & 1 & 55 & 21 & 0 \\
\hline & & & 2 & 66 & 10 & 36 \\
\hline \multirow{2}{*}{$\begin{array}{l}\text { TRANSYT-7F with } \\
\text { HC }\end{array}$} & \multirow{2}{*}{8.18} & \multirow{2}{*}{78} & 1 & 55 & 23 & 0 \\
\hline & & & 2 & 68 & 10 & 0 \\
\hline \multirow{2}{*}{$\begin{array}{l}\text { TRANSYT-7F with } \\
\text { GA }\end{array}$} & \multirow{2}{*}{8.17} & \multirow{2}{*}{79} & 1 & 58 & 21 & 0 \\
\hline & & & 2 & 69 & 10 & 6 \\
\hline
\end{tabular}

Table 2. The best $P$ I and signal timings for two junction road network

While the best $P I$ is 8.18 in TRANSYT-7F with HC, the best PI is 8.17 in TRANSYT-7F with GA. The common network cycle time is $79 \mathrm{sec}$ and $78 \mathrm{sec}$ in TRANSYT-7F with GA and HC. As can be seen in Table 2, the PI obtained from the ACOTRANS model is slightly better than the values obtained from the TRANSYT-7F with GA and HC. These results indicate that the ACOTRANS produces comparable results to the in TRANSYT-7F with GA and HC. Hence, the proposed ACOTRANS model provides an alternative to the HC and GA optimization algorithm in TRANSYT-7F that could produce better results in terms of the PI for this small sized network.

In order to test the ACOTRANS model's effectiveness and robustness, it is also applied to medium sized road network. The network is illustrated based upon the one used by [23]. Basic layouts of the network and stage configurations are given in Fig. (7) and (8). This network includes 23 links and 21 signal setting variables at six signalcontrolled junctions. 


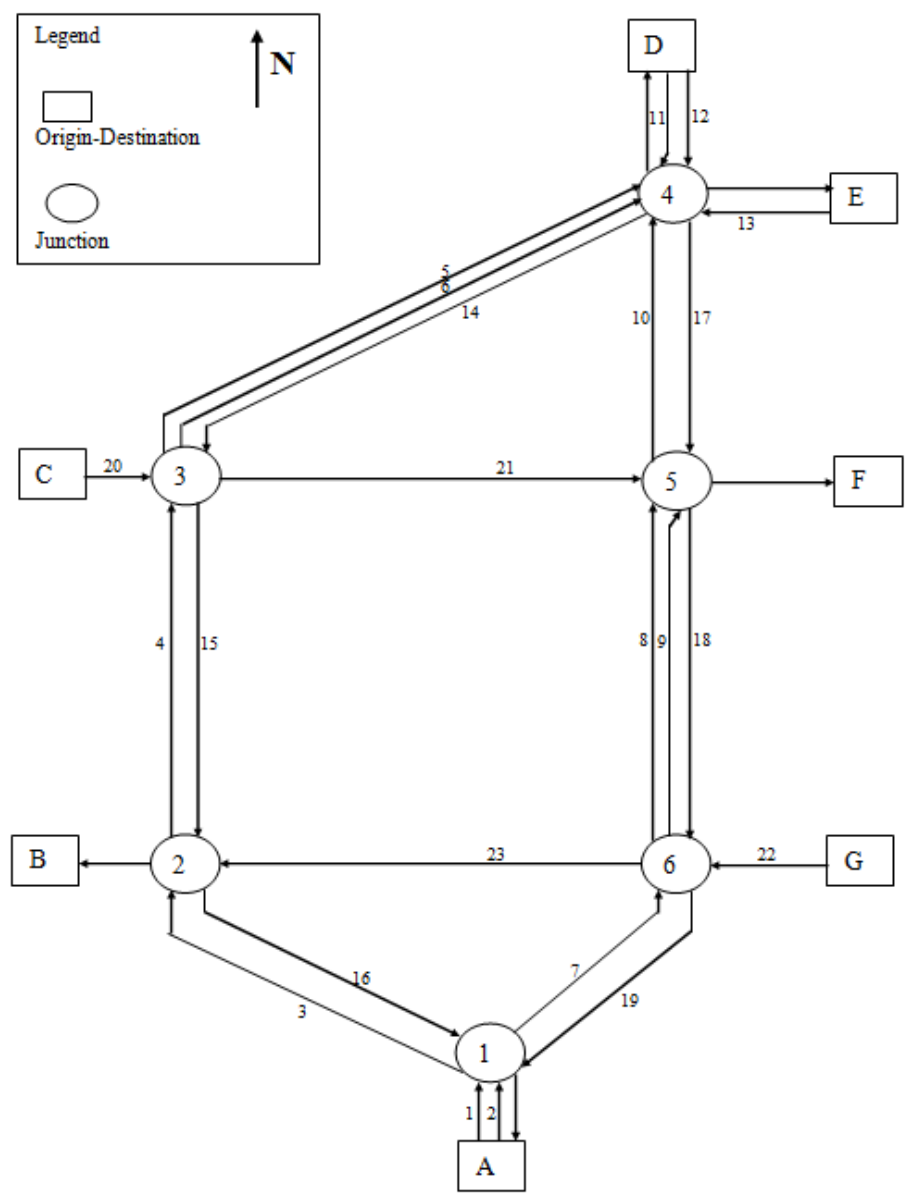

Figure 7. Layout for medium sized network 


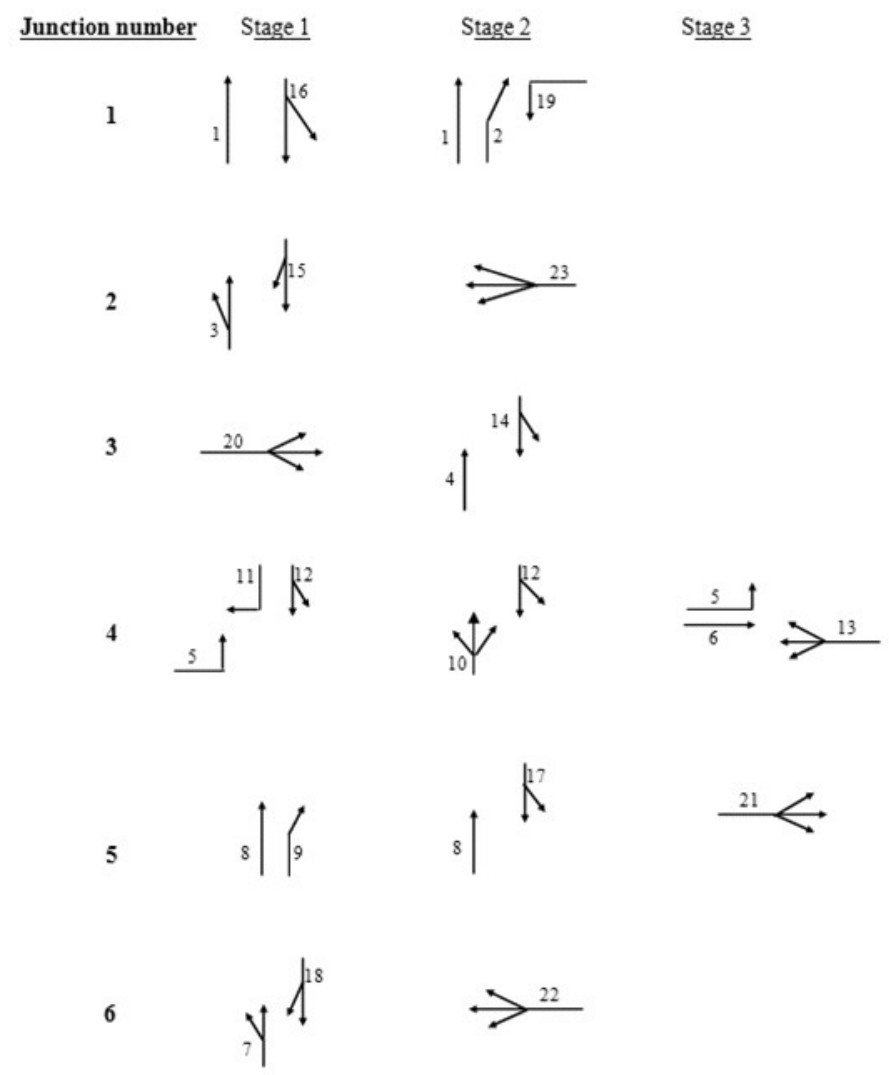

Figure 8. Stage configurations for medium sized network

The fixed set of link flows, taken from [22], is given in Table 3.

\begin{tabular}{cccc}
\hline $\begin{array}{c}\text { Link } \\
\text { number }\end{array}$ & $\begin{array}{c}\text { Link } \\
\text { flow } \\
\text { (veh/h) }\end{array}$ & $\begin{array}{c}\text { Saturation flow } \\
\text { (veh/h) }\end{array}$ & $\begin{array}{c}\text { Free-flow } \\
\text { travel time (sec) }\end{array}$ \\
\hline 1 & 716 & 2000 & 1 \\
\hline 2 & 463 & 1600 & 1 \\
\hline 3 & 716 & 3200 & 10 \\
\hline 4 & 569 & 3200 & 15 \\
\hline 5 & 636 & 1800 & 20 \\
\hline 6 & 173 & 1850 & 20 \\
\hline 7 & 462 & 1800 & 10 \\
\hline
\end{tabular}




\begin{tabular}{|c|c|c|c|}
\hline $\begin{array}{c}\text { Link } \\
\text { number }\end{array}$ & $\begin{array}{c}\text { Link } \\
\text { flow } \\
\text { (veh/h) }\end{array}$ & $\begin{array}{c}\text { Saturation flow } \\
\text { (veh/h) }\end{array}$ & $\begin{array}{c}\text { Free-flow } \\
\text { travel time (sec) }\end{array}$ \\
\hline 8 & 478 & 1850 & 15 \\
\hline 9 & 120 & 1700 & 15 \\
\hline 10 & 479 & 2200 & 10 \\
\hline 11 & 499 & 2000 & 1 \\
\hline 12 & 250 & 1800 & 1 \\
\hline 13 & 450 & 2200 & 1 \\
\hline 14 & 789 & 3200 & 20 \\
\hline 15 & 790 & 2600 & 15 \\
\hline 16 & 663 & 2900 & 10 \\
\hline 17 & 409 & 1700 & 10 \\
\hline 18 & 350 & 1700 & 15 \\
\hline 19 & 625 & 1500 & 10 \\
\hline 20 & 1290 & 2800 & 1 \\
\hline 21 & 1057 & 3200 & 15 \\
\hline 22 & 1250 & 3600 & 1 \\
\hline 23 & 837 & 3200 & 15 \\
\hline
\end{tabular}

Table 3. Fixed set of link flows on medium sized network

The constraints on signal timings are set as follows:

$36 \leq c \leq 140$ cycle time constraint

$0 \leq \theta \leq c$ offsets

$7 \leq \varphi \leq$ cgreen split

$I_{1-2}=I_{2-1}=5$ seconds intergreen time

In Fig. (9), the convergence of the ACOTRANS for medium sized network can be seen. The best signal timings obtained from the previous cycle are stored in order not to being bad local optimum. By means of the generated new ant colony, global optimum is searched around the best signal setting parameters using reduced search space during the algorithm process. As shown Fig. 9, the ACORSES starts the solution process according to random generated signal timings and it was found that the value of PI is about 551. The ACORSES keeps the best solution and then it uses the best solution to the optimum in the reduced search space. Optimum solution is then searched in the reduced search space during the algorithm progress. The significant improvement on the objective function takes place in the first few cycle because the ACORSES starts with randomly generated ants in a large colony size. After that, small improvements to the objective function takes place since the pheromone updating rule and new created ant colony provide new solution vectors on the differ- 
ent search directions. Finally, the minimum number of PI reached to the value of about 362 after 150 cycles.

This numerical test shows that the ACORSES is able to prevent being trapped in bad local optimum for solving ATC problem. In order to overcome non-convexity, the ACORSES starts with a large base of solutions, each of which provided that the solution converges to the optimum and it also uses the reduced search space technique. In ACORSES, new ant colony is created according to randomly generated $\alpha$ value. For this reason, any of the newly created solution vectors may be outside the reduced search space. Therefore, created new ant colony prevents being trapped in bad local optimum. The ACORSES is able to achieve global optimum or near global optimum to optimise signal timings because it uses concurrently the reduce search technique and the orientation of all ants to the global optimum.

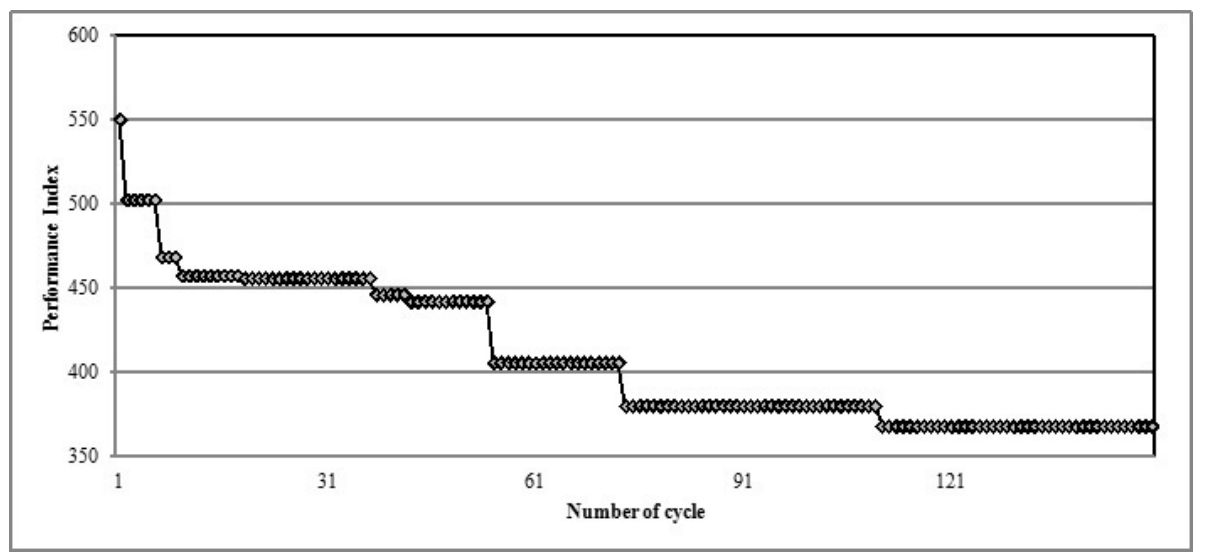

Figure 9. The convergence of the ACOTRANS for medium sized network

The common network cycle time obtained from the ACOTRANS is $106 \mathrm{sec}$. Moreover, medium sized network is optimized using TRANSYT-7F, which are GA and HC optimization tools. For studied network, the ACOTRANS and TRANSYT-7F optimizers' results are given in Table 4 . The best PI is found as 410.0 in TRANSYT-7F with GA while its value is obtained as 420.5 in TRANSYT-7F with HC. The common network cycle time is 114 sec and $120 \mathrm{sec}$ in TRANSYT-7F with HC and GA, respectively. The ACOTRANS improves network's PI $11.7 \%$ and $13.9 \%$ when it is compared with TRANSYT-7F with GA and HC. It also decreases common cycle time $11.5 \%$ and $7 \%$ when it is compared with the cycle times produced TRANSYT-7F with GA and HC. These results showed that the ACOTRANS model illustrates good performance for optimizing traffic signal timings in coordinated networks with fixed set of link flows. Hence, the ACOTRANS provides an alternative to the HC and GA optimization tools in TRANSYT-7F that could produce better results in terms of $P I$. 


\begin{tabular}{|c|c|c|c|c|c|c|c|}
\hline & \multirow[b]{2}{*}{$P I$} & \multirow{2}{*}{$\begin{array}{l}\text { Cycle } \\
\text { Time } \\
\text { c(s) }\end{array}$} & \multirow{2}{*}{$\begin{array}{c}\text { Junction } \\
\text { number } \\
i\end{array}$} & \multicolumn{3}{|c|}{ Duration of stages (s) } & \multirow{2}{*}{$\begin{array}{c}\text { Offsets } \\
\text { (s) } \\
7 \leq \varphi \leq c\end{array}$} \\
\hline & & & & $\begin{array}{c}\text { Stage } 1 \\
I_{1-2}=I_{2-1}=5\end{array}$ & $\begin{array}{c}\text { Stage } 2 \\
\theta_{i}\end{array}$ & $\begin{array}{c}\text { Stage } 3 \\
\varphi_{i, 1}\end{array}$ & \\
\hline \multirow{6}{*}{ ACOTRANS } & \multirow{6}{*}{361.9} & \multirow{6}{*}{106} & 1 & 46 & 60 & - & 0 \\
\hline & & & 2 & 64 & 42 & - & 96 \\
\hline & & & 3 & 62 & 44 & - & 10 \\
\hline & & & 4 & 38 & 34 & 34 & 36 \\
\hline & & & 5 & 15 & 33 & 58 & 38 \\
\hline & & & 6 & 34 & 72 & - & 74 \\
\hline \multirow{6}{*}{ TRANSYT-7F with HC } & \multirow{6}{*}{420.5} & \multirow{6}{*}{114} & 1 & 44 & 70 & - & 0 \\
\hline & & & 2 & 56 & 58 & - & 98 \\
\hline & & & 3 & 69 & 45 & - & 98 \\
\hline & & & 4 & 43 & 36 & 35 & 98 \\
\hline & & & 5 & 15 & 36 & 63 & 98 \\
\hline & & & 6 & 39 & 75 & - & 98 \\
\hline \multirow{6}{*}{ TRANSYT-7F with GA } & \multirow{6}{*}{410.0} & \multirow{6}{*}{120} & 1 & 60 & 60 & - & 0 \\
\hline & & & 2 & 74 & 46 & - & 89 \\
\hline & & & 3 & 71 & 49 & - & 37 \\
\hline & & & 4 & 44 & 38 & 38 & 106 \\
\hline & & & 5 & 15 & 38 & 67 & 75 \\
\hline & & & 6 & 60 & 60 & - & 55 \\
\hline
\end{tabular}

Table 4. The results for medium sized network

\section{Conclusions}

This study deals with the area traffic control problem using the ACOTRANS. For this purpose, ACO based algorithm called ACORSES was used. The ACORSES algorithm for solving ATC problem differs from approaches in that new ant colony is generated at each cycle with the assistance of the best solution of the previous information. Moreover, the best solution that is obtained from the previous evaluation is saved to prevent being trapped in bad local optimum. The ACOTRANS is introduced to optimize traffic signal timings at coordinated signalized network. TRANSYT-7F is used to compute PI for a given set of signal timing and staging plan in network. The ACOTRANS is tested on two road networks in order to show its robustness and effectiveness. For first test network which contains two junctions, results showed that the ACOTRANS produces slightly better results than TRANSYT-7F with GA and HC. Proposed algorithm was also applied to medium sized network which 
contains six junctions. Results also showed that the ACOTRANS improves network's PI by $11.7 \%$ and $13.9 \%$ according to TRANSYT-7F with GA and HC. The ACOTRANS provides an alternative to the HC and GA optimization tools in TRANSYT-7F that could produce better results in terms of the PI. As a result, the ACOTRANS may be used to optimize traffic signal timings at coordinated signalized network. In future works, the ACOTRANS will be applied to a real-sized network in order to demonstrate the applicability and the effectiveness of the proposed model.

\section{Author details}

Soner Haldenbilen*, Ozgur Baskan and Cenk Ozan

*Address all correspondence to: shaldenbilen@pau.edu.tr

Pamukkale University, Engineering Faculty, Department of Civil Engineering, Transportation Division, Turkey

\section{References}

[1] Teklu, F., Sumalee, A., \& Watling, D. (2007). A genetic algorithm approach for optimizing traffic control signals considering routing. Computer-Aided Civil and Infrastructure Engineering, 22, 31-43.

[2] Webster, F. V. (1958). Traffic Signal Settings Road Research Technical Paper. HMSO London [39].

[3] Robertson, DI. (1969). TRANSYT' method for area traffic control. Traffic Engineering and Control, 10, 276-81.

[4] TRANSYT-7F Release 11.3 Users Guide,. (2008). McTrans Center, University of Florida, Gaineville, Florida.

[5] Baskan, O., Haldenbilen, S., Ceylan, H., \& Ceylan, H. (2009). A new solution algorithm for improving performance of ant colony optimization. Applied Mathematics and Computation, 211(1), 75-84.

[6] Wong, SC. (1995). Derivatives of the performance index for the traffic model from TRANSYT. Transportation Research Part B, 29(5), 303-327.

[7] Wong, SC. (1996). Group-based optimisation of signal timings using the TRANSYT traffic model. Transportation Research Part B, 30(3), 217-244.

[8] Heydecker, BG. (1996). A decomposed approach for signal optimization in road networks. Transportation Research Part B, 30(2), 99-114. 
[9] Wong, S. C., Wong, W. T., Xu, J., \& Tong, C. O. (2000). A Time-dependent TRANSYT Traffic Model for Area Traffic Control. Proceedings of the Second International Conference on Transportation and Traffic Studies. ICTTS, 578-585.

[10] Wong, S. C., Wong, W. T., Leung, C. M., \& Tong, C. O. (2002). Group-based optimization of a time-dependent TRANSYT traffic model for area traffic control. Transportation Research Part B, 36, 291-312.

[11] Girianna, M., \& Benekohal, R. F. (2002). Application of Genetic Algorithms to Generate Optimum Signal Coordination for Congested Networks. Proceedings of the Seventh International Conference on Applications of Advanced Technologies in Transportation, 762-769.

[12] Ceylan, H. (2006). Developing Combined Genetic Algorithm-Hill-Climbing Optimization Method for Area Traffic Control. Journal of Transportation Engineering, 132(8), 663-671.

[13] Chen, J., \& Xu, L. (2006). Road-Junction Traffic Signal Timing Optimization by an adaptive Particle Swarm Algorithm. 9th International Conference On Control, Automation, Robotics And Vision, 1- 5, 1103-1109.

[14] Chiou, S-W. (2007). A hybrid optimization algorithm for area traffic control problem. Journal of the Operational Research Society, 58, 816-823.

[15] Chiou, S. W. (2007). An efficient computation algorithm for area traffic control problem with link capacity expansions. Applied Mathematics and Computation, 188, 1094-1102.

[16] Dan, C., \& Xiaohong, G. (2008). Study on Intelligent Control of Traffic Signal of Urban Area and Microscopic Simulation. Proceedings of the Eighth International Conference of Chinese Logistics and Transportation Professionals, Logistics: The Emerging Frontiers of Transportation and Development in China, 4597-4604.

[17] Li, Z. (2011). Modeling Arterial Signal Optimization with Enhanced Cell Transmission Formulations. Journal of Transportation Engineering, 137(7), 445-454.

[18] Dorigo, M., Di Caro, G., \& Gambardella, L. M. (1999). Ant Algorithms for Discrete Optimization. Artificial Life, MIT press.

[19] Eshghi, K., \& Kazemi, M. (1999). Ant colony algorithm for the shortest loop design problem. Computers \& Industrial Engineering, 50, 358-366.

[20] Baskan, O., \& Haldenbilen, S. (2011). Ant Colony Optimization Approach for Optimizing Traffic Signal Timings. Ant Colony Optimization- Methods and Applications, InTech, 205-220.

[21] Ceylan, H., \& Bell, M. G. H. (2004). Traffic signal timing optimisation based on genetic algorithm approach, including drivers' routing. Transportation Research Part B, 38(4), 329-342. 
[22] Ceylan, H. (2002). A genetic algorithm approach to the equilibrium network design problem. Ph.D. Thesis, University of Newcastle upon Tyne, UK.

[23] Allsop, R. E., \& Charlesworth, J. A. (1977). Traffic in a signal-controlled road network: an example of different signal timings including different routings. Traffic Engineering Control, 18(5), 262-264. 
